\title{
Fatal Pulmonary Hemorrhage Through Fiberoptic Broncoscopy in a Well Controlled Diabetic Female With Mucormycosis
}

\author{
${ }^{1}$ P. Di Carlo, ${ }^{2}$ D. Cabibi, ${ }^{1}$ A. Mazzola, ${ }^{3}$ D. De Luca, ${ }^{3}$ A.M. La Rocca and ${ }^{3}$ E. Sacco \\ ${ }^{1}$ Istituto Patologia Infettiva e Virologia, Università degli studi di Palermo, Italy \\ ${ }^{2}$ Istituto di Anatomia Patologica, Facoltà di Medicina, Università degli Studi di Palermo, Italy \\ ${ }^{3}$ UO Malattie dell' Apparato Respiratorio V, Azienda Ospedaliera V. Cervello, Palermo, Italy
}

\begin{abstract}
A rare case of invasive pulmonary mucormycosis complicated by fatal massive haemoptysis through bronchoscopy in a patient with a well controlled diabetes is reported. Histological features of infection are reported and the characteristic aspects of the staining are discussed. Fiberoptic bronchoscopy revealed bilateral obstruction of the segmental bronchi and fatal massive bleeding occurring because of biopsy sampling. Caution is warranted in the use of bronchoscopy sampling in patients with radiological features of cavitated lesions of fungal origins. Better preventive and early therapeutic antifungal treatments are needed to avoid this serious complication.
\end{abstract}

Key words: Pulmonary haemorrhage, diabetis, fiberoptic bronchoscopy, mucormycosis

\section{INTRODUCTION}

The term zygomycosis refers to infections caused by fungi belonging to a group called Zygomycetes, which includes the genera Rhizopus, Absidia and Rhizomисоr. These were previously assigned to the genus mucor and considered responsible for the disease known as mucormycosis ${ }^{[1,2]}$. These fungi are ubiquitous and are common inhabitants of decomposing matter. In contrast to their widespread distribution, these fungi can cause serious and rapidly fatal infections, particularly in the immuno-compromised, such as poorly controlled diabetics with ketoacidosis ${ }^{[3-5]}$.

The fungi invade major blood vessels, leading to extensive necrosis and in the case of the extensive pulmonary disease, bleeding occurs into the lungs because of biopsy or suction. The principal risk factors are profound $(\mathrm{PN}<500$ per $\mathrm{mm} 3)$ and prolonged neutropenia, perturbed phagocyte function and cellular immune deficiency (AIDS, immunosuppressive treatment in organ and bone marrow recipients) ${ }^{[3-6]}$. Patients with diabetes mellitus can also develop pulmonary mucormycosis with less fulminant course but with atypical presentation of unique nodule ${ }^{[7]}$.

Bronchial biopsy (surgical or transbronchial) of abnormal tissue, broncho-aspirated or BAL by bronchoscopy with microbiologic evaluation are the most efficient methods for diagnosis ${ }^{[3-4]}$.

We report a rare case of diffuse pulmonary mucormycosis in a well-controlled diabetic female with fatal pulmonary haemorrhage during fiberoptic bronchoscopy procedure.

\section{CASE REPORT}

A 62-year-old woman living in the city of Palermo, Italy, with a history of fever and a persistent cough from three weeks, was admitted to V. Cervello hospital to perform a planned fiberoptic bronchoscopy (FB) to assess the nature of diffuse pulmonary lesion evidenced in CT chest scan (Fig. 1).

The instrumental findings were diagnosed as multiple cavitated lesions of lungs, some containing air and with a hyperdense capsule in the bronchi consistent with bronchiectasis and others mimicking heteroplastic cavitary lesions (Fig. 1).

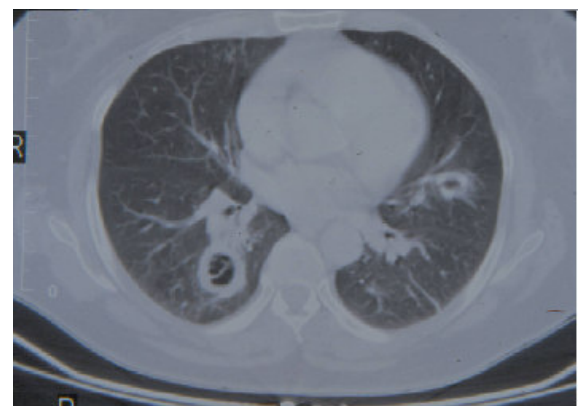

Fig. 1: Transverse TC scan showing circular masses in pulmonary fields

Corresponding Author: Daniela Cabibi, Dipartimento di Patologia Umana, Università degli Studi, Palermo Via del Vespro, 129 Palermo Tel: +39 0916553533 Fax: +39 0916553549 
The patient had no significant clinical history except for diabetes mellitus controlled with oral antidiabetic treatment and a suitable diet.

At the time of hospitalization, the patient's white blood cell count was $10 \times 10^{9} \mathrm{~L}^{-1}$ with $50 \%$ neutrophils, haemoglobin and blood glucose was $13.0 \mathrm{~g}$ and $260 \mathrm{mg}$ $100 \mathrm{~mL}^{-1}$ respectively. There were no coagulation alterations and thrombocyte count was 200.000 cells $/ \mathrm{mL}$.

The bronchoscopy examination revealed mucous and necrotic plug full occluding the lingula bronchus and the apical segment of both lower lobe bronchus (B6) Fig. 2A. Grasping forceps were used to remove the necrotic occluding plug for cyto-histological analysis and other samples were obtained through bronchial aspiration. At once these sampling procedures were drawn, the patients had a massive haemorrhage following by cardiorespiratory arrest and subsequent admission in ICU in state of coma.

The histological examination showed large areas of necrosis of bronchial mucosa, with peripheral granulomatous reaction and several multi-nucleated cells (Fig. 2B). In the necrotic areas numerous broad, very rarely septate, haphazardly branched hyphae were evident. Noteworthy, the hyphae were deeply stained by hematoxylin-eosin (H\&E), better than by special stains for fungi, such as PAS (Fig. 2C-D) and Gomori methenamine silver stain (GMS)

The morphological aspects and the stain affinity suggested the they were consistent with mucormycosis . This hypothesis was confirmed by the culture of the the bronchial aspirate sample (istological sample) showing filamentous mycetes belonging to the class Zigomycetes.

On the basis of this data, the patient was treated with $5 \mathrm{mg}$ of liposomal amphotericin $\mathrm{B} /$ body weight.

No evidence of disseminate infection was found. and the analysis of cerebrospinal liquor not showed any cerebral mycoses. The patients died 20 days later recovering in ICU.

\section{DISCUSSION}

As our knowledge diffuse pulmonary mucormycosis are rarely observed in well controlled diabetic patients and the massive haemorrhage occurring in bronchoscopic procedure induced us to record this fatal case.

Diabetes mellitus is one of the main underlying conditions behind the cases of pulmonary mucormycosis. In our patient's case, there was no evidence of severe or persistent hyperglycemia and the general condition and absence of coagulation alterations indicated that a bronchoscopy examination could be carried out.

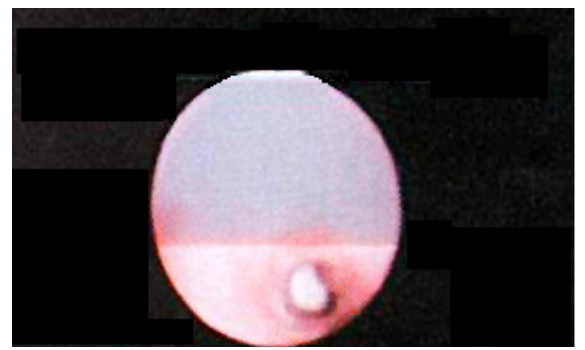

Fig. 2A: Broncoscopy imaging showing muco-necrotic tissue in the right hemisystem

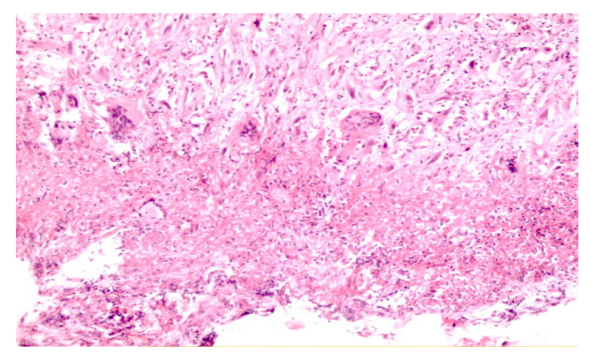

Fig. 2B: Large areas of necrosis with peripheral granulomatous reaction and several multinucleated cells (Hematoxylin-eosin; overall magnification 100x)

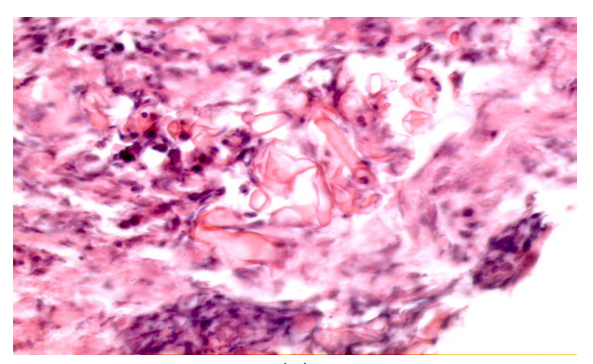

(c)

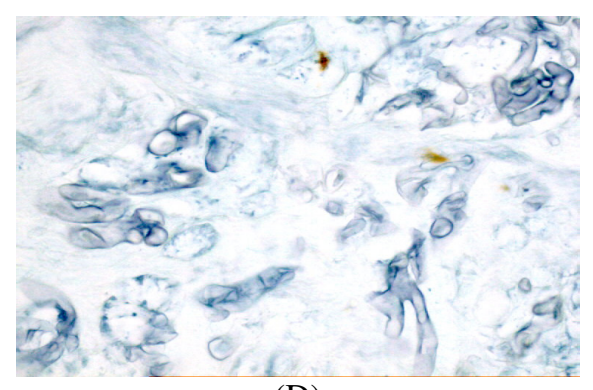

(D)

Fig. C-D) In the necrotic areas numerous broad, very rarely septate, haphazardaly branched hyphae were evident. The hyphae were deeply stained by hematoxylin-eosin staining (C), better than by PAS staining (D) 
Fiberoptic bronchoscopy with biopsy and/ or bronchial aspirate is needed to confirm a diagnosis of pulmonary mucormycosis, as radiological findings are non-specific and may suggest other diseases or different fungal aetiology such as aspergillosis.

In the histological and cytological samples, the hyphae are well evidenced on the routinely performed H\&E slides, but this stain should always be confirmed with a more fungus-specific tissue stain such as GMS or PAS. Nevertheless, these fungus specific staining may be less intense than would be seen with other hyaline fungi ${ }^{[2]}$ and mucor is sometimes better evidenced in $\mathrm{H} \& \mathrm{E}$ stain.

In mucorales endobronchial form, the vascular involvement of great vessels may be a cause of spontaneous fatal haemoptysis. Several cases are described especially in leukemia patients although in most of described cases no patient showed relevant thrombocytopenia or coagulative abnormalities ${ }^{[5]}$.

In our case, the fatal hemorrhagic event occurred in the course of standardized bronchoscopic procedures performed to get some specimens for histological and microbiological assessment .

Fiberoptic bronchoscopic examination is a useful procedure for identifying bronchial obstructions and endoluminal lesions, as well as for assessing the tracheobronchial tree beyond stenoses. Moreover, the procedure makes it possible to restore normal airflow in areas around the blockage which are airless (possible atelectasis or subatelectasis). Al Majed reported the removal of mucormycosis lesion through a rigid bronchoscope $\mathrm{e}^{[9]}$.

The endobronchial mucous and necrotic plugs seen in our presented case were related to the vascular invasion of the pulmonary vessels caused by mucoraceous hyphae $e^{[1,2]}$.

The technique of sampling in broncoscopy examination may be the trigger to vessel rupture with massive haemorrhage into a conducting bronchus causing asphyxiation.

Although the risk related to bronchoscopy procedure are well known especially in unclear or well definite diagnosis, particularly caution is warranted in the use of endoscopic examen in patients with radiological features suggestive of fungal infection of order Mucorales. In these cases, the only bronchoalveolar lavage has been advocated as less invasive technique. Recently, non invasive technique such as virtual broncoscopy appeared to be useful for assessing the friability of lesion in cavitated disease ${ }^{[9]}$.

In a previous report, the development of the air crescent sign on chest radiograph was suggested as an important clinical indicator of potentially fatal haemoptysis ${ }^{[10]}$. This finding with the features of changes and progression of lesion should lead to early antifugal and surgical therapy ${ }^{[11]}$.

In our case the unique radiological findings didn't suggest to start early adequate antifungal or surgical treatment. Without a sure diagnosis, only early treatment seems to be the best hope of reducing the serious morbidity associated with this disseminate pulmonary form. The majority of patients were treated with lipid preparations of amphotericin (predominantly liposomal) with few reactions or adverse events ${ }^{[12]}$. For years, amphotericin B has been the drug of choice for these highly aggressive infections. Recently in unresponsive patients to monotherapy with liposomal amphotericin B a favourably response was observed following the addition of the echinocandin caspofungin acetate $^{[13]}$. Of the newly introduced second-generation triazoles, voriconazole is not active against the Zygomycetes, while posaconazole has demonstrated activity in vitro, in animal models and in case reports $^{[14]}$.

\section{REFERENCES}

1. Ellis, D.H., 2005. Systemic zygomycosis. In: Microbiology and microbial infections, medical mycology. Merz, W.G. and R.J. Hay, (Eds.). Topley and Wilson's, 10th Edn., pp: 659-686. Hodder Arnold, London, United Kingdom.

2. Ribes, J.A., C.L. Vanover-Sams and D.J. Baker, 2000. Zygomycetes in human disease. Clin. Microbiol. Rev., 13 (2): 236-301.

3. Freifeld, A.G. and P.C. Iwen, 2004. Zygormycosis. Semin Respir Crit Care Med., 25: 221-31.

4. Prabhu and R. Patel, 2004. Mucormycosis and entomophthoramycosis: A review of the clinical manifestations, diagnosis and treatment. Clin. Microbiol. Infect., 10 (Suppl 1): 31-47.

5. Pagano, L., P. Ricci, A. Nosari, A. Tonso, M. Buelli, M. Montillo, L. Cudillo, A. Cenacchi, C. Savignana and L. Melillo, 1995. Fatal haemoptysis in pulmonary filamentous mycosis: An underevaluated cause of death in patients with acute leukaemia in haematological complete remission. A retrospective study and review of the literature. Gimema Infection Program. Br. J. Haematol., 89 (3): 500-505.

6. Gale, A.M. and W.P. Kleitsch, 1972. Solitari pulmonary nodule due to phycomycosis (mucormycosis). Chest, 62: 752-755.

7. Passamonte, P.M. and J.D. Dix, 1985. Nosocomial pulmonary mucormycosis with fatal massive hemoptysis. Am. J. Med. Sci., 289: 65-67. 
8. Al-Majed S., F. Al-Kassimi, M. Ashour, M.O. Mekki and S. Sadiq, 1992. Removal of endobronchial mucormycosis lesion through a rigid bronchoscope. Thorax; 47 (3): 203-204.

9. Finkelstein, Steven E. Summers, M. Ronald, Nguyen, M. Dao, I.V. Stewart, H. John, Tretler, A. Jean, et al. 2002. Virtual bronchoscopy for evaluation of malignant tumors of the thorax. J. Thoracic Cardiovascular Surgery. 123(5): 967-972.

10. Dykhuizen, R.S., K.N. Kerr and R.L. Soutar Air, 1994. Crescent sign and fatal haemoptysis in pulmonary mucormycosis. Scand. J. Infect. Dis., 26 (4): 498-501.

11. Harada, M., T. Manabe, K. Yamashita and N. Okamoto 1992. Pulmonary mucormycosis with fatal massive hemoptysis. Acta Pathol Jap. 42 (1): 49-55.
12. Chopra, 2002. AmBisome in the treatment of fungal infections: The UK experience. J. Antimicrob Chemother., 49 (Suppl) 1: 43-47.

13. Vazquez, L., J.J. Mateos, C. Sanz-Rodriguez, E. Perez, D. Caballero and J.F. San Miguel, 2005. Successful treatment of rhinocerebral zygomycosis with a combination of caspofungin and liposomal amphotericin B. Haematologica. 90 (12 Suppl): ECR39.

14. Page, R.L., J. Schwiesow and A. Hiltsm, 2007. Posaconazole as salvage therapy in a patient with disseminated zygomycosis: Case Report and Rev. of the Literature. Pharmacotherapy, 27 (2): 290-298. 